\title{
Commentary: A Skeptical View of Experimental Gene Therapy to Block Epileptogenesis
}

\author{
F. Edward Dudek \\ Department of Physiology, University of Utah School of Medicine, Salt Lake City, Utah 84108
}

\begin{abstract}
Summary: Gene therapy offers exciting new options for treating epileptic seizures, and even for blocking the development of epilepsy (i.e., epileptogenesis) after a brain insult. Although the available studies provide interesting new data, the experiments discussed in this issue also have limitations and raise concerns. The criticisms offered in this commentary center around the nature of the experimental testing (e.g., changes in seizure threshold), the animal models (e.g., kindling), and the measures of epileptogenesis in those animal models with spontaneous seizures (e.g., the latent period after pilocarpine-in-
\end{abstract}

duced status epilepticus). Another set of criticisms relate to the relative lack of positive controls showing that the actual mechanism purported to be activated via the gene-therapeutic approach has in fact been upregulated in the specific animals that show the hypothetical antiepileptic result. This commentary takes the con side in the debate, to generate constructive criticism to help direct future studies to provide increasingly stronger data to support the view that gene therapy approaches may be useful in the treatment of epilepsy. Key Words: Epilepsy, seizures, status epilepticus, kindling, seizure threshold.

\section{INTRODUCTION}

Although gene therapy has considerable potential as a new class of treatments for intractable epilepsy, the experimental data supporting this approach are open to several possible criticisms. The aim of this brief commentary is to identify those criticisms and concerns, with the goal of developing stronger experimental support for the potential usefulness of gene therapy for treatment of intractable epilepsy. These comments may also apply to experimental investigations on other types of antiepileptic therapies.

\section{THE DIFFERENT APPROACHES FOR STUDYING ALTERATIONS IN EPILEPTOGENESIS}

\section{Convulsive seizure threshold}

Several of the studies using gene therapy and other techniques or drugs to block or suppress epileptogenesis essentially measure seizure threshold as an endpoint of effectiveness, which is then assumed to be inversely proportional to the susceptibility to epileptic seizures.

Address correspondence and reprint requests to: F. Edward Dudek, Ph.D., Department of Physiology, University of Utah School of Medicine, 420 Chipeta Way, Suite 1700, Salt Lake City, UT 84108. E-mail: ed.dudek@hsc.utah.edu.
With this technique, kainic acid or some other convulsive substance is injected into control and experimental animals, and the time from injection to the first seizure (i.e., latency) is used as a measure of seizure threshold. The latency for controls ranges from a few tens of minutes ${ }^{1}$ to a few hours ${ }^{2}$; thus, the duration of the latency is quite variable across animals and experiments.

In our experience over many years with kainate and pilocarpine injections in otherwise normal animals, the latency to the first seizure is extremely variable and depends on exactly how the injections are performed and the seizures are recorded (e.g., electrographic versus behavioral). In some studies with this technique, the number of animals in the different groups has been relatively small (as few as 5 and often $<8$ ), which can be problematic, given the high variance with this technique. A major determinant of latency is the time for the drug (e.g., kainic acid) to diffuse through a tortuous pathway from the injection site to the brain area or areas where the seizures actually start. In animals that are already having seizures, the seizure threshold is also likely a function of time since the last seizure or seizures. Thus, the use of this technique to detect a hypothetical change in seizure susceptibility is highly susceptible to errors and is indirect, at best.

Animal models of acquired epilepsy that are based on the technique of measuring seizure threshold to injection 
of a convulsive drug have generally not been viewed as robust or easily reproducible. Some studies have used electrical stimulation instead of convulsant drugs as an assay to determine the threshold for induction of seizures, and although the variance with electrical stimulation is typically less than the latency-to-seizure after convulsant drug treatment, this technique can also be quite variable. The technique of seizure-threshold testing, regardless of how it is done, seems particularly indirect and susceptible to bias. Furthermore, although epilepsy may be viewed to be a decrease in seizure threshold, induction of a change in seizure threshold is not exactly the same as altering epileptogenesis. Therefore, the use of measurements of seizure threshold after a gene therapy protocol does not realistically reflect chronic epilepsy, and is also fraught with problems of bias and high variance relative to the number of animals used in most of these studies.

\section{Kindling: effects on seizures versus epileptogenesis}

A variety of studies using cell or gene therapy and various pharmacological agents (e.g., NMDA receptor antagonists) have reported a suppression of kindling. ${ }^{3}$ One of the primary problems with most of these studies is that the treatment also suppresses individual stimulation-evoked seizures during kindling, thus changing the severity of the insult that actually induces the epileptogenesis. The observation that a hypothetical therapy suppresses the rate of kindling, when that therapeutic approach also suppresses the seizures to individual stimuli, is therefore weak evidence for an antiepileptogenic effect (as opposed to an antiseizure effect), because the rate of kindling depends on the robustness of the evoked seizures. That is, if the therapy reduces individual electrically evoked seizures, it would also be expected to suppress the rate of kindling even if the therapy is not antiepileptogenic. In conclusion, those studies that show a suppression of kindling, but also have an effect on evoked seizures, do not provide evidence that the potential therapy suppresses epileptogenesis per se (i.e., as opposed to seizures).

\section{Animal models with spontaneous recurrent seizures} (i.e., epilepsy)

Status epilepticus. Although many animal models of acquired epilepsy are available, those models based on chemoconvulsant or electrically induced status epilepticus are the most widely employed, because they are relatively easy to use and because they develop spontaneous recurrent seizures and typically involve a latent period (i.e., a delay from the end of the status epilepticus to the first motor seizure and the onset of chronic epilepsy). Although some aspects of the experimental design of studies based on epileptogenesis after status epilepticus may be stronger than results that depend on measurements of seizure threshold or kindling in terms of demonstrating a true antiepileptogenic, or even a disease-modifying effect, ${ }^{4}$ these studies also raise several concerns.

For example, Raol et al. ${ }^{5}$ reported that treatment of the rats with an adeno-associated virus (AAV) vector that induces expression of altered $\mathrm{GABA}_{\mathrm{A}}$ receptor subunits $\left(\mathrm{AAV}-\mathrm{GABA}_{\mathrm{A}}\right)$ leads to a longer latent period and a reduction in the number of animals that had been observed to have spontaneous recurrent seizures by 30 days. In those studies, however, status epilepticus was induced 2 weeks after the injection of an AAV-GABA vector and a sham treatment, and Raol et al. ${ }^{5}$ reported that the pilocarpine-induced status epilepticus was not altered. Note, however, that only some of the animals in the control $(n=4)$ and experimental AAV-GABA $(n=$ 7) groups were actually implanted for electrographic recording, and thus the analysis of status epilepticus and the subsequent seizures was based primarily if not exclusively on behavioral seizures.

Similarly, Raol et al. ${ }^{5}$ reported that the amount of diazepam required to block the status epilepticus was unaltered-but diazepam is unlikely to block electrographic seizure activity when administered 60 min after the onset of pilocarpine-induced status epilepticus (see Lehmkuhle et al. ${ }^{6}$ ), even when diazepam suppresses the behavioral seizures; therefore, these data do not provide strong evidence that the pretreatment with AAV-GABA did not suppress the status epilepticus. The pretreatment with AAV-GABA $A_{A}$ vector could have reduced the electrographic activity during the pilocarpine-induced status epilepticus, which would not have been detected in these studies but could account for the apparent disease-modifying results. Thus, experimental designs that include protocols that could alter the intensity of the status epilepticus may appear to modify or even block epileptogenesis when in fact they suppress the actual insult that is thought to be responsible for the subsequent epilepsy.

Latent period. The duration of the latent period (i.e., the time to either the first electrographic or motor seizure) has traditionally been considered to be a measure of epileptogenesis. Raol et al. ${ }^{5}$ reported that the $\mathrm{AAV}-\mathrm{GABA}_{\mathrm{A}}$ treatment led to a longer latent period after pilocarpine-induced status epilepticus. In other models based on status epilepticus, the initial epileptic or late seizures (i.e., after the latent period) in most animals are nonconvulsive, with minimal behavioral manifestations, ${ }^{7-9}$ and the critical measure regarding the initial seizures several days after status epilepticus is likely to be electrographic activity. In addition, even though the latent period has traditionally been the cornerstone concept representing epileptogenesis, use of the latent period in antiepileptogenesis studies has both conceptual and practical flaws.

Recent work (see also earlier studies of Bertram and Cornett $^{7,8}$ ) with continuous electrographic recordings in 
the repeated, low-dose kainate model indicates that a slow, continuous but variable increase in seizure frequency (i.e., with seizure clusters) characterizes the early phases of epileptogenesis (see next section), and the latent period may simply be the first of many long interseizure intervals during the early stages of epileptogenesis. ${ }^{9}$ The conceptual and practical problem is that the measurement of the latent period is essentially the determination of an asymptotic departure of increased seizure frequency from a baseline, which is theoretically and mathematically problematic. Thus, if epileptogenesis is a slowly developing and continuous process, then a more appropriate and accurate measure of epileptogenesis is the determination of the rate of increase of seizure frequency over time. Nonetheless, if the status epilepticus in the two groups (i.e., control without AAV-GABA and experimental group with $\mathrm{AAV}-\mathrm{GABA}_{\mathrm{A}}$ ) was actually identical, then the available data do suggest that the gene therapy treatment alters the development of epilepsy (i.e., causes disease modification).

Seizure frequency. In a recent study by Noé et al., ${ }^{10}$ an AAV viral vector that increased the expression of neuropeptide $\mathrm{Y}$ in the hippocampus (AAV-NPY) reduced the frequency of spontaneous recurrent seizures compared to controls when administered $>3$ months after induction of status epilepticus. Seizure frequency was analyzed over periods of 2 weeks before and 4 weeks after administration of the AAV-NPY, and was compared to similar recordings from animals that were sham controls and those that received empty AAV. This approach of assessing changes in seizure frequency after gene therapy is clearly more rigorous and realistic than in other previous studies. Although these data appear promising, one concern (also expressed by the authors) is that the amount of monitoring-even though more than in many other studies in this field-was still limited. Virtually all of the animals were in a slow-growth phase (see Williams et al. ${ }^{9}$ ), with low baseline seizure rates and limited increases in seizure frequency during the study.

The data in Figure 2 of Noé et al. ${ }^{10}$ show that the animals treated with AAV-NPY had an overall lower seizure rate than the two control groups. Nonetheless, as expected, the data in this figure suggest considerable variability within and across animals (compare controls and empty-virus groups), and raises the question: how different were the AAV-NPY animals from the two control groups? The data in Figure 2 and Table 1 of Noé et al. ${ }^{10}$ suggest that the main effect of the AAV-NPY animals compared to the two control groups was to reduce the variance by decreasing the number of days with relatively high seizure rates, so that the seizure frequency of the empty-AAV control group after injection was one or a few seizures per day, whereas the experimental AAV-NPY group had between one seizure per day to one seizure every few days; therefore, the baseline and post-treatment seizure rate was quite low and variable, and the differences between groups represent relatively few seizures in relation to the amount of recording time, leading to the authors' statement that the "unequivocal demonstration of this hypothesis requires a longer follow-up study of spontaneous seizures."

Furthermore, it is unknown whether temporary suppression of seizures, independent of an effect on any other possible antiepileptogenic mechanism, slows the progressive increase in seizure frequency (i.e., do seizures beget seizures? and does a therapy-induced decrease in seizures cause a transient decrease in seizures?). Therefore, if AAV-NPY acutely suppresses seizures, it may secondarily reduce the progressive increase in seizure frequency after status epilepticus. Noé et al. ${ }^{10}$ also emphasize the need for further studies showing that learning, memory, and other brain functions are not adversely affected by the AAV-NPY. In conclusion, although the data are not without caveats and concerns, these studies provide the most comprehensive and encouraging data to date that a gene therapy approach suppresses spontaneous recurrent seizures and may actually be antiepileptogenic (or at least, disease modifying).

\section{THE VALUE OF OPTIMAL POSITIVE CONTROLS}

At least two main approaches have been outlined in gene therapy and cell therapy studies. One approach has been aimed at altering the GABA system (e.g., GABA receptors) in a manner that would hypothetically suppress epileptogenesis. The cell therapy approaches have typically involved increasing the number of GABAergic interneurons through some type of stem cell technique (see review by Thompson ${ }^{11}$ ), but the endpoint would be augmentation of GABAergic transmission. A second approach involves viral-mediated increase in neuropeptide (e.g., NPY or galanin) expression in selective hippocampal neurons. In both cases, little or no data have actually been provided for an electrophysiological change in the hippocampal network. Although various studies may show some evidence of increased gene expression, these studies do not show clear electrophysiological changes of an antiepileptogenic nature in slices from animals containing the altered system.

For example, it would be extremely useful in the animals in which the $\mathrm{GABA}_{\mathrm{A}}$ receptors have been altered to show that GABAergic transmission is increased. Specifically, does the use of the gene therapy approach in Raol et al. ${ }^{5}$ lead to miniature inhibitory postsynaptic currents of larger amplitude? In a cell therapy approach that aims to increase the number of GABAergic interneurons, one should be able to show an increase in the frequency of miniature inhibitory postsynaptic currents 
in the experimental animals versus the controls. Similarly, the logic of using increased NPY expression is that NPY suppresses excitatory glutamatergic transmission. What is needed is to show in epileptic animals with reduced seizures, or blocked epileptogenesis, that NPY release is increased and that this leads to a suppression of glutamatergic transmission, compared to controls. These types of electrophysiological data would provide a positive control, showing that the hypothetical mechanism thought to mediate antiepileptogenic therapy is actually operative in the tissue, which would greatly strengthen the support for these hypotheses. Furthermore, one would expect that those animals showing the greatest alteration from the therapy might actually have the most reduced epileptogenesis, but this has not been clearly shown.

\section{CONCLUSIONS}

This commentary aims to provide constructive criticism of the studies aimed at using gene therapy (and potentially other antiepileptic therapies) to suppress seizures and epileptogenesis (as discussed and reviewed elsewhere in this issue). The first family of criticisms is that relatively little of the data actually show a suppression of chronic epilepsy, and the data that do purport to show this call for some caveats. Second, the studies typically lack strong positive controls, where the experimental animals with a gene therapy-induced alteration in gene expression have been shown to demonstrate the predicted functional alteration (e.g., altered GABAergic or glutamatergic transmission). Finally, it is not clear why these gene therapy approaches will be more efficacious and have fewer adverse effects than traditional antiepileptic drug medication. A recent review has outlined the strengths of gene therapy approaches to treating epilepsy, ${ }^{12}$ but the authors did not directly address the concerns and criticisms raised here. Another series of studies with these gene therapy tools, and with more quantitative and extensive monitoring, preferably by independent investigators, could provide new and stronger data to support this approach before trials are initiated on human patients with intractable epilepsy.

Acknowledgments: I would like to thank Anthony van den Pol and H. Steve White for reading the manuscript and for making several valuable suggestions. The research in our laboratory related to the issues discussed in this commentary has been supported by the National Institute of Neurological Disorders and Stroke, National Institutes of Health (NS16683, NS045144, NS049620, and NS42359).

\section{REFERENCES}

1. Lin EJD, Richichi C, Young D, Baer K, Vezzani A, During MJ. Recombinant AAV-mediated expression of galanin in rat hippocampus suppresses seizure development. Eur J Neurosci 2003; 18:2087-2092.

2. Foti S, Haberman RP, Samulski RJ, McCown TJ. Adeno-associated virus-mediated expression and constitutive secretion of NPY or NPY13-36 suppresses seizure activity in vivo. Gene Ther 2007; 14:1534-1536.

3. Richichi C, Lin EJD, Stefanin D, et al. Anticonvulsant and antiepileptogenic effects mediated by adeno-associated virus vector neuropeptide $\mathrm{Y}$ expression in the rat hippocampus. J Neurosci 2004;24:3051-3059.

4. Walker MC, White HS, Sander JWAS. Disease modification in partial epilepsy. Brain 2002;125:1937-1950.

5. Raol YH, Lund IV, Bandyopadhyay S, et al. Enhancing GABA $_{A}$ receptor $\alpha 1$ subunit levels in hippocampal dentate gyrus inhibits epilepsy development in an animal model of temporal lobe epilepsy. J Neurosci 2006;26:11342-11346.

6. Lehmkuhle MJ, Thomson KE, Scheerlinck P, Pouliot W, Greger B, Dudek FE. A simple quantitative method for analyzing electrographic status epilepticus in rats. J Neurophysiol 2009 Jan. 7 (Epub ahead of print)

7. Bertram EH, Cornett J. The ontogeny of seizures in a rat model of limbic epilepsy: evidence for a kindling process in the development of chronic spontaneous seizures. Brain Res 1993;625:295300.

8. Bertram EH, Cornett JF. The evolution of a rat model of chronic spontaneous limbic seizures. Brain Res 1994;661:157-162.

9. Williams PA, White AM, Clark S, Ferraro DJ, Swiercz W, Staley KJ, Dudek FE. Development of spontaneous recurrent seizures after kainate-induced status epilepticus. J Neurosci 2009;29:21032122.

10. Noè F, Pool AH, Nissinen J, et al. Neuropeptide $Y$ gene therapy decreases chronic spontaneous seizures in a rat model of temporal lobe epilepsy. Brain 2008;131:1506-1515.

11. Thompson K. Transplantation of GABA-producing cells for seizure control in models of temporal lobe epilepsy. Neurotherapeutics 2009;6:284-294.

12. Riban V, Fitzsimons HL, During MJ. Gene therapy in epilepsy. Epilepsia 2009;50:24-32. 